\title{
A Combined Experimental and Computational Study of Dihydrido- Phosphinooxazoline-Iridium Complexes
}

\author{
Clément Mazet, Sebastian P. Smidt, Markus Meuwly* and Andreas Pfaltz* \\ Contribution from the Department of Organic Chemistry, St. Johanns Ring 19, and the \\ Department of Physical Chemistry, Klingelbergstrasse 80, Basel University, CH-4056 \\ Basel (Switzerland).
}

\section{Supporting Information}

General procedure for the hydrogenation of $\operatorname{Ir}(\mathrm{PHOX})$ complexes at the NMR scale: In a glove-box, the iridium complex was weighed into an NMR tube, which was then filled with deuterated solvent under an argon atmosphere and put in a long narrow Schlenk tube sealed with a rubber septum. The sample was then thermostated at the desired temperature. A cooling mantle filled with dry-ice was placed around the upper part of the Schlenk in order to avoid evaporation of the deuterated solvent. Hydrogen gas (quality $>99.99 \%$ ) was introduced through a long stainless-steel needle. The needle was attached to a three-way stop-cock allowing to purge the whole apparatus by three argonvacuum cycles before switching to hydrogen. The gas was bubbled through the solution at flow rates between 2 to $5 \mathrm{~mL} / \mathrm{min}$. The NMR tube was finally sealed with a rubber septum and introduced into the NMR spectrometer (Bruker Avance 500) pre-cooled to the desired temperature.

Representative ${ }^{1} \mathrm{H}$ and ${ }^{31} \mathrm{P}$ NMR data for complexes C-1, C-2 and D-2:

\begin{tabular}{|c|c|c|c|c|}
\hline hydrido-Ir complex & ${ }^{1} \mathrm{H} \quad \delta(\mathrm{ppm})$ & ${ }^{31} \mathbf{P}\left\{{ }^{1} \mathrm{H}\right\} \delta(\mathrm{ppm})$ & ${ }^{2} \boldsymbol{J}(\mathbf{H}, \mathbf{H})$ & ${ }^{2} \boldsymbol{J}(\mathbf{H}, \mathbf{P})$ \\
\hline $\mathrm{C}-1$ & $-12.7-15.6$ & 4.9 & $6.8 \mathrm{~Hz}$ & $20.3 \mathrm{~Hz}$ \\
\hline C-2 & $-17.3-29.5$ & 6.8 & $7.0 \mathrm{~Hz}$ & $18.7 \mathrm{~Hz}$ \\
\hline D-2 & $-17.6-29.8$ & 7.4 & $7.0 \mathrm{~Hz}$ & $18.7 \mathrm{~Hz}$ \\
\hline
\end{tabular}


${ }^{1} \mathrm{H}$ Spectrum of $[(\mathrm{PHOX}) \operatorname{Ir}(\mathrm{COD})] \mathrm{BAr}_{\underline{E}}\left[(\mathrm{PHOX}) \operatorname{Ir}(\mathrm{H})_{2}(\mathrm{COD})\right] \mathrm{BAr}_{\underline{F}} \underline{\mathbf{C}-\mathbf{1}}$ $\left(500 \mathrm{MHz}\right.$; [D8]-THF) at $-40^{\circ} \mathrm{C}$
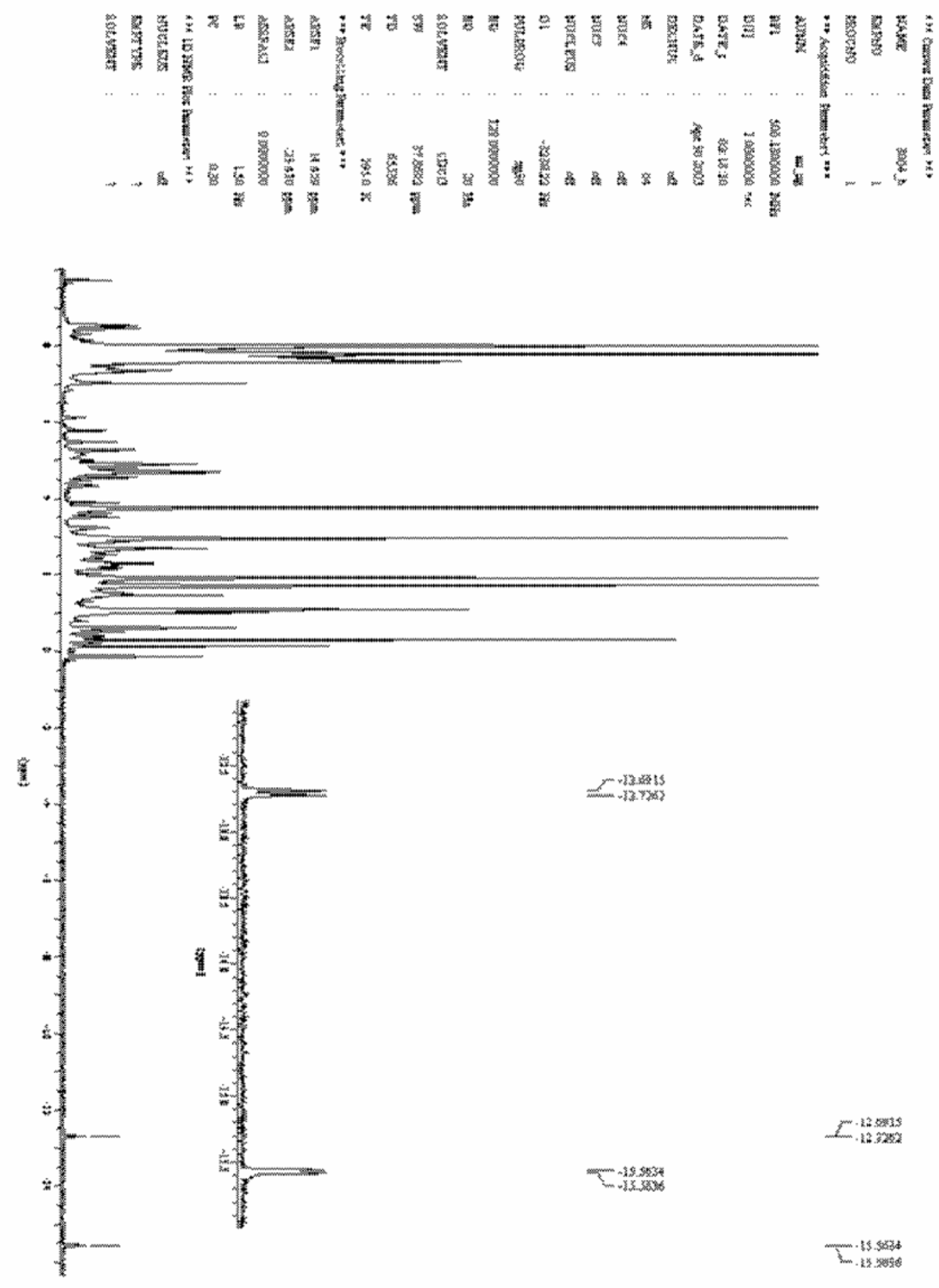


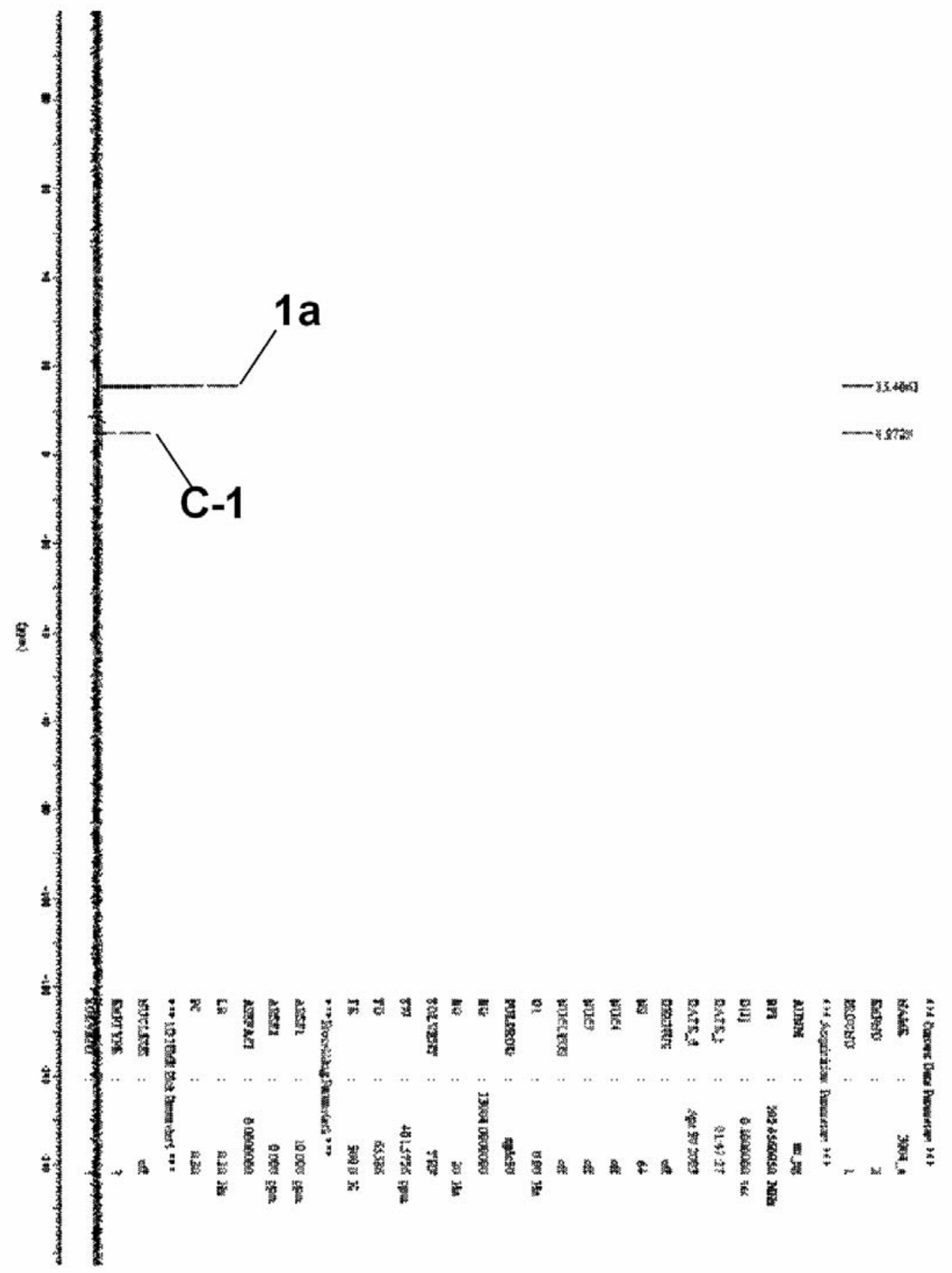




\section{${ }^{1} \mathrm{H}$ and ${ }^{31} \mathrm{P}\left\{{ }^{1} \mathrm{H}\right\}$ NMR data of $\left[(\mathrm{PHOX}) \operatorname{Ir}(\mathrm{H})_{2}(\mathrm{COD})\right] \mathrm{BAr}_{\mathrm{F}} \underline{\mathbf{C}-\mathbf{1}}$}

$(500 \mathrm{MHz} ;[\mathrm{D} 8]-\mathrm{THF})$ at $-40^{\circ} \mathrm{C}$

${ }^{1}$ H NMR (500 MHz, [D8]-THF): $\delta(\mathrm{ppm}) 8.45$ (m, 2Ḥ $\left.\mathrm{H}_{\mathrm{Ar}}\right), 8.20-7.35\left(\mathrm{~m}, 12 \mathrm{H}, \mathrm{H}_{\mathrm{Ar}}\right)$ among which: $7.82\left(1 \mathrm{H}, \mathrm{H}_{\mathrm{Ar} \text { ortho }}=\mathrm{H} 6\right), 7.52\left(1 \mathrm{H}, \mathrm{H}_{\mathrm{Ar} \text { ortho }}=\mathrm{H} 5\right), 5.78\left(\mathrm{bs}, 1 \mathrm{H}, \mathrm{CH}_{\mathrm{ol} \mathrm{COD}}\right.$ $=\mathrm{H} 4), 5.51$ (bs, $\left.1 \mathrm{H}, \mathrm{CH}_{\mathrm{ol} \mathrm{COD}}\right), 5.25$ (bs, $\left.1 \mathrm{H}, \mathrm{CH}_{\mathrm{ol} \mathrm{COD}}\right), 5.10-4.90\left(\mathrm{~m}, 1 \mathrm{H}, \mathrm{H}_{\text {oxa }}\right), 4.80-$ $4.70\left(\mathrm{~m}, 2 \mathrm{H}, \mathrm{H}_{\text {oxa }}\right), 4.72$ (bs, 1H, $\left.\mathrm{CH}_{\mathrm{ol} \mathrm{COD}}=\mathrm{H} 8\right), 3.93\left(\mathrm{~m}, 1 \mathrm{H}, \mathrm{CH}_{2} \mathrm{COD}\right), 2.81(\mathrm{~m}, 1 \mathrm{H}$, $\mathrm{CH}_{2} \mathrm{COD}$ ), 2.68 ( $\mathrm{m}, 1 \mathrm{H}, \mathrm{CH}_{2}$ COD $\left.=\mathrm{H} 7\right), 2.63$ ( $\mathrm{m}, 1 \mathrm{H}, \mathrm{CH}_{2} \mathrm{COD}$ ), 2.60 (m, 1H, $\mathrm{CH}_{2} \mathrm{COD}$ ), $2.30\left(\mathrm{~m}, 1 \mathrm{H}, \mathrm{CH}_{2} \mathrm{COD}\right), 2.29\left(\mathrm{~m}, 1 \mathrm{H}, \mathrm{CH}_{2} \mathrm{COD}\right), 1.95\left(\mathrm{~m}, 1 \mathrm{H}, \mathrm{CH}_{2} \mathrm{COD}\right), 2.22(\mathrm{~m}, 1 \mathrm{H}$, $\left.\mathrm{CH}_{\mathrm{iPr}}=\mathrm{H} 3\right), 1.00\left(\mathrm{~d}, 3 \mathrm{H}, \mathrm{CH}_{3} \mathrm{iPr}\right),-0.15\left(\mathrm{~d}, 3 \mathrm{H}, \mathrm{CH}_{3} \mathrm{iPr}\right),-12.7\left(\mathrm{dd}, 1 \mathrm{H}, \mathrm{H}_{\text {hydride }}=\mathrm{H} 1\right)$, $15.6\left(\mathrm{dd}, 1 \mathrm{H}, \mathrm{H}_{\text {hydride }}=\mathrm{H} 2\right)$.

${ }^{31} \mathbf{P}\left\{{ }^{1} \mathrm{H}\right\}$ NMR (202.5 MHz, [D8]-THF): $\delta$ (ppm) 4.9.

*) Identification of protons $\mathrm{H} 5$ and $\mathrm{H} 6$ (in ortho position on the two phenyl substituents of the $\mathrm{P}$-atom) is based on a ${ }^{1} \mathrm{H}\left\{{ }^{31} \mathrm{P}\right\}$ NMR spectra.

*) A NOE map of the representative interactions between hydrides and the ligand backbone as well as a three-dimensional representation of isomer $\mathbf{C - 1}$ are depicted on the next page. 

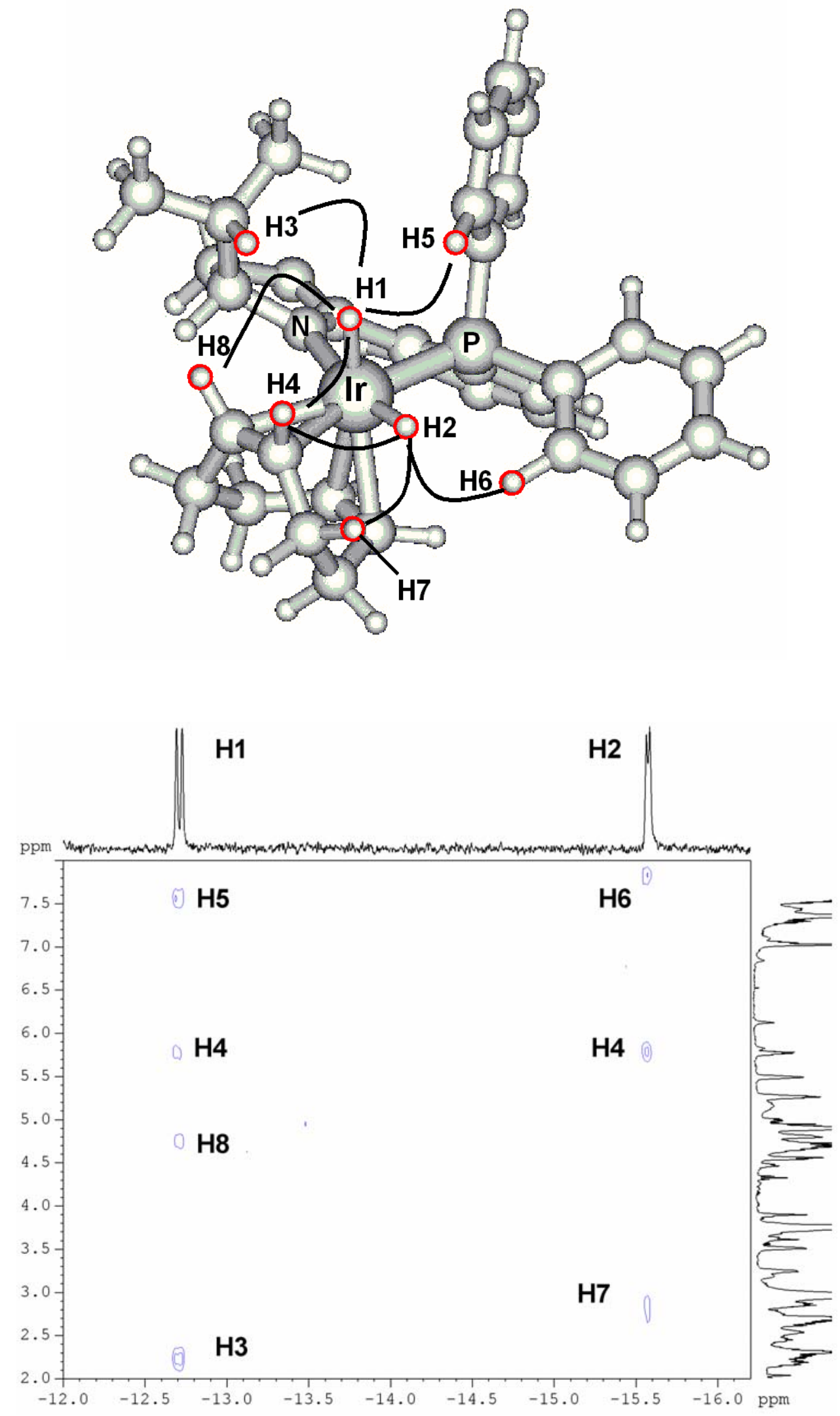
$\underline{{ }^{1} \mathrm{H} \text { and }{ }^{31} \mathrm{P}\left\{{ }^{1} \mathrm{H}\right\} \text { NMR data of }\left[(\mathrm{PHOX}) \operatorname{Ir}(\mathrm{H})_{2}(\text { solvent })_{2}\right] \mathrm{BAr}_{\mathrm{E}}(\mathbf{C}-2 \text { \& D-2 })}$

$\left(500 \mathrm{MHz} ;\right.$ [D8]-THF) at $0^{\circ} \mathrm{C}$

C-2: ${ }^{1} \mathbf{H}$ NMR (500 MHz, [D8]-THF): $\delta(\mathrm{ppm}) 8.35\left(\mathrm{~m}, 2 \mathrm{H} \mathrm{H}_{\mathrm{Ar}}\right), 7.80-6.90(\mathrm{~m}, 12 \mathrm{H}$, $\left.\mathrm{H}_{\mathrm{Ar}}\right), 4.79-4.43\left(\mathrm{~m}, 3 \mathrm{H}, \mathrm{H}_{\mathrm{oxa}}\right), 2.33\left(\mathrm{~m}, 1 \mathrm{H}, \mathrm{CH}_{\mathrm{iPr}}\right), 0.90$ (d, 3H, $\left.\mathrm{CH}_{3} \mathrm{iPr}\right), 0.35$ (d, 3H, $\mathrm{CH}_{3}$ iPr), -17.3 (dd, 1H, $\mathrm{H}_{\text {hydride }}$ ), -29.5 (dd, $1 \mathrm{H}, \mathrm{H}_{\text {hydride) }}$;

${ }^{31} \mathbf{P}\left\{{ }^{1} \mathrm{H}\right\}$ NMR (202.5 MHz, [D8]-THF): $\delta$ (ppm) 6.8 .

D-2: ${ }^{1}$ H NMR (500 MHz, [D8]-THF): $\delta(\mathrm{ppm}) 8.15$ (m, 2H $\left.\mathrm{H}_{\mathrm{Ar}}\right), 7.80-6.90(\mathrm{~m}, 12 \mathrm{H}$, $\mathrm{H}_{\mathrm{Ar}}$ ), 4.79-4.3 (m, 3H, $\mathrm{H}_{\text {oxa }}$ ), 3.81 (bd, 1H, $\left.\mathrm{CH}_{\mathrm{ol} \mathrm{COD}}\right), 2.18$ ? (m, 1H, $\left.\mathrm{CH}_{\mathrm{iPr}}\right), 0.89$ (d, 3H, $\left.\mathrm{CH}_{3} \mathrm{PPr}\right), 0.39$ (d, 3H, $\mathrm{CH}_{3 \mathrm{iPr}}$ ), -17.6 (dd, $1 \mathrm{H}, \mathrm{H}_{\text {hydride }}$ ), -29.8 (dd, $1 \mathrm{H}, \mathrm{H}_{\text {hydride }}$ );

${ }^{31} \mathbf{P}\left\{{ }^{1} \mathbf{H}\right\}$ NMR (202.5 MHz, [D8]-THF): $\delta(\mathrm{ppm}) 7.4$. 
$\underline{\left.{ }^{1} \mathrm{H} \text { spectra of }\left[(\mathrm{PHOX}) \operatorname{Ir}(\mathrm{H})_{2}{ }_{2} \text { (solvent }\right)_{2}\right] \mathrm{BAr}_{\underline{F}}(\mathbf{C}-\mathbf{2} \& \mathbf{D}-2)}$

$\underline{(500 \mathrm{MHz} ; \text { [D8]-THF }) \text { at } 0^{\circ} \mathrm{C}(\delta \text { from }-1 \text { to } 5.5 \mathrm{ppm})}$

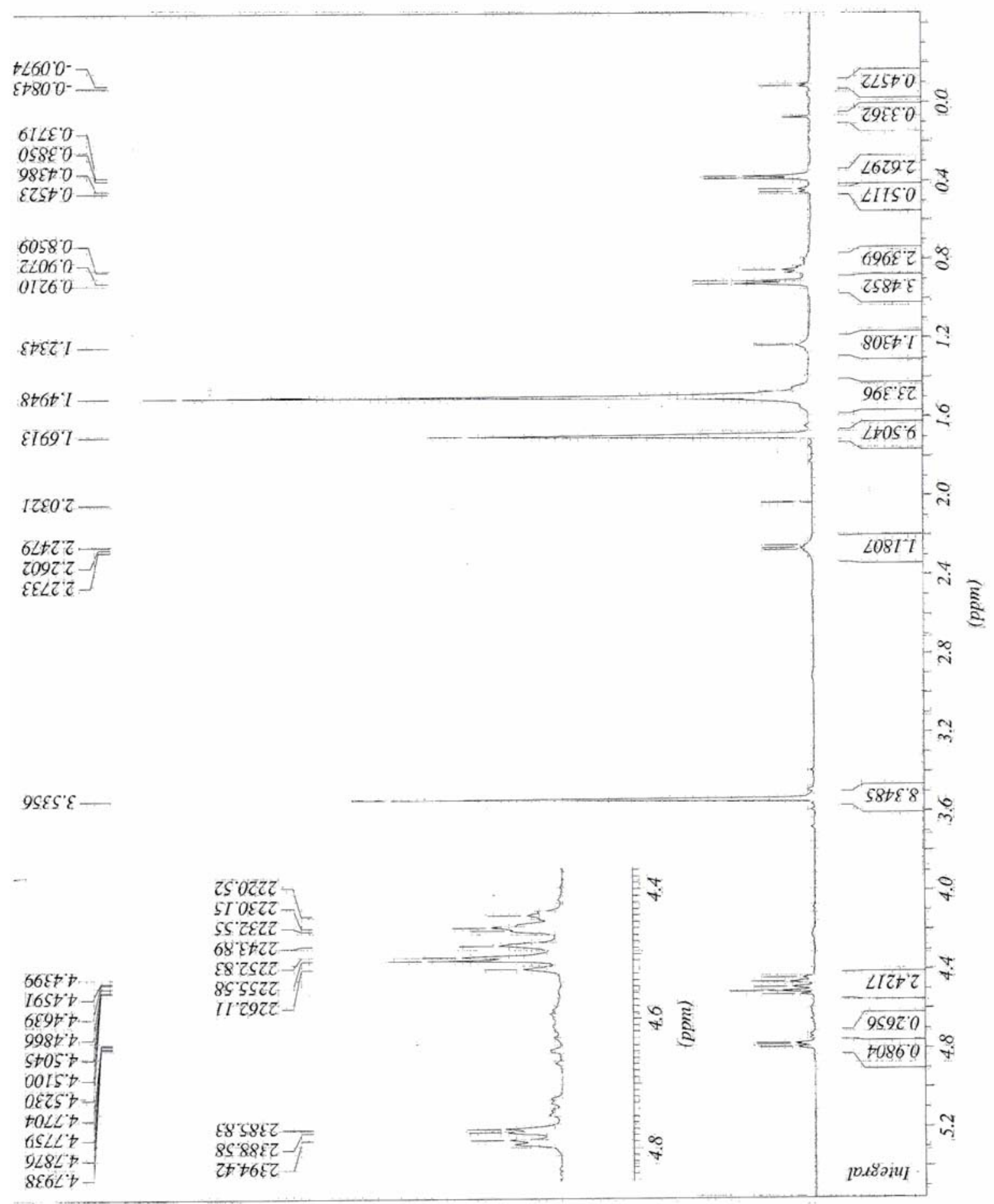


${ }^{\left.{ }_{1}^{1} \mathrm{H} \text { spectra of }\left[(\mathrm{PHOX}) \operatorname{Ir}(\mathrm{H})_{2} \text { (solvent }\right)_{2}\right] \mathrm{BAr}_{\underline{E}}(\mathbf{C}-\mathbf{2} \& \mathbf{D}-2)}$

(500 MHz; [D8]-THF) at $0^{\circ} \mathrm{C}$ ( $\delta$ from -1 to $\left.5.5 \mathrm{ppm}\right)$

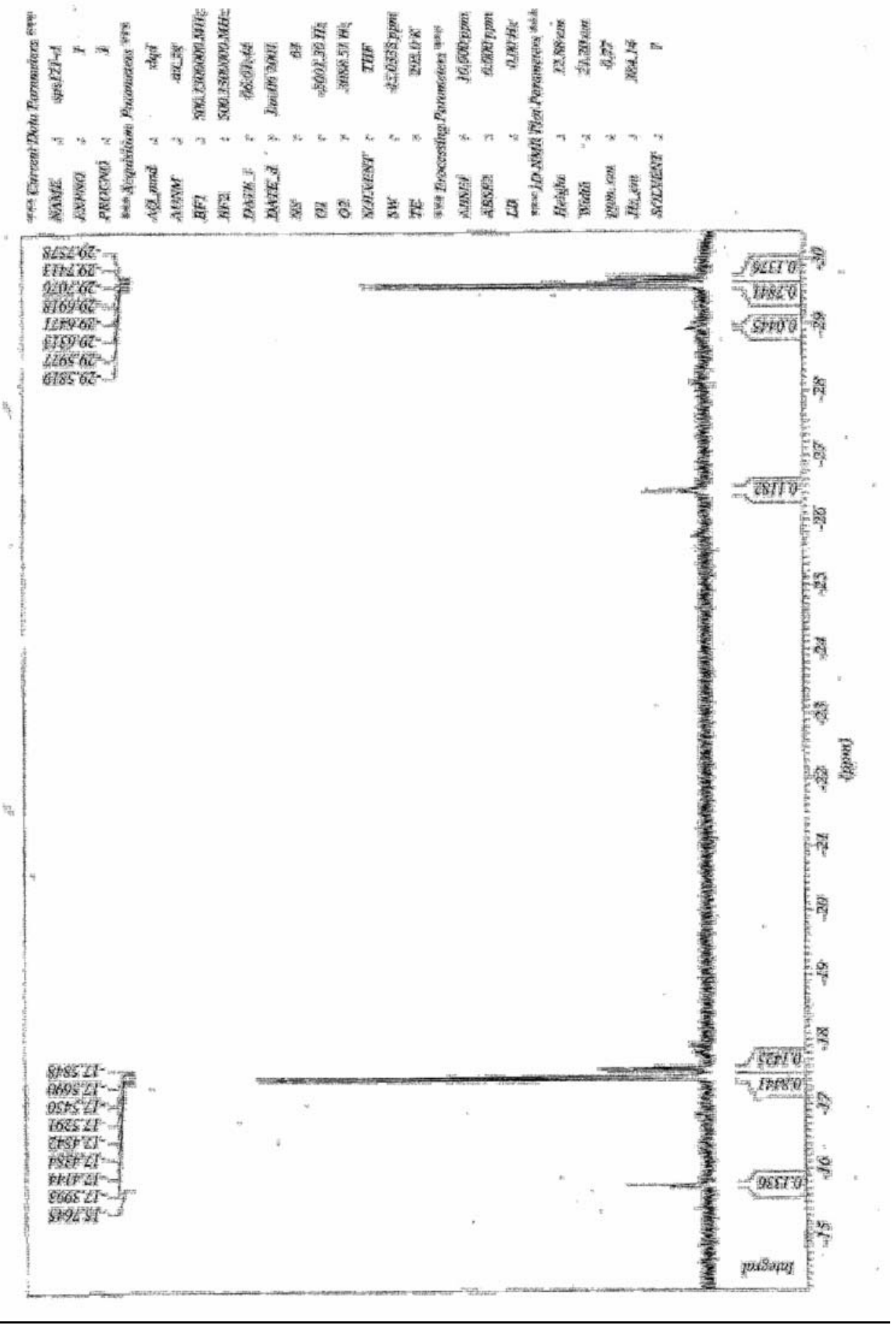


*) Discrimination between isomer C-2 and D-2 is based on the observed NOE contact between the apical hydride $(\delta=-29.5 \mathrm{ppm})$ and the proton of the iso-propyl substituent $(\delta$ $=2.33 \mathrm{ppm})$ of the oxazoline ring.

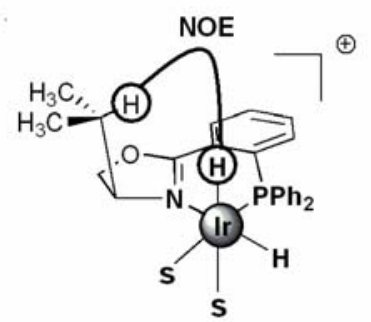

C-2

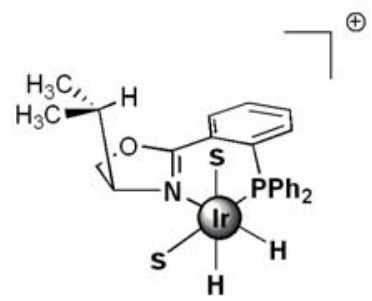

D-2

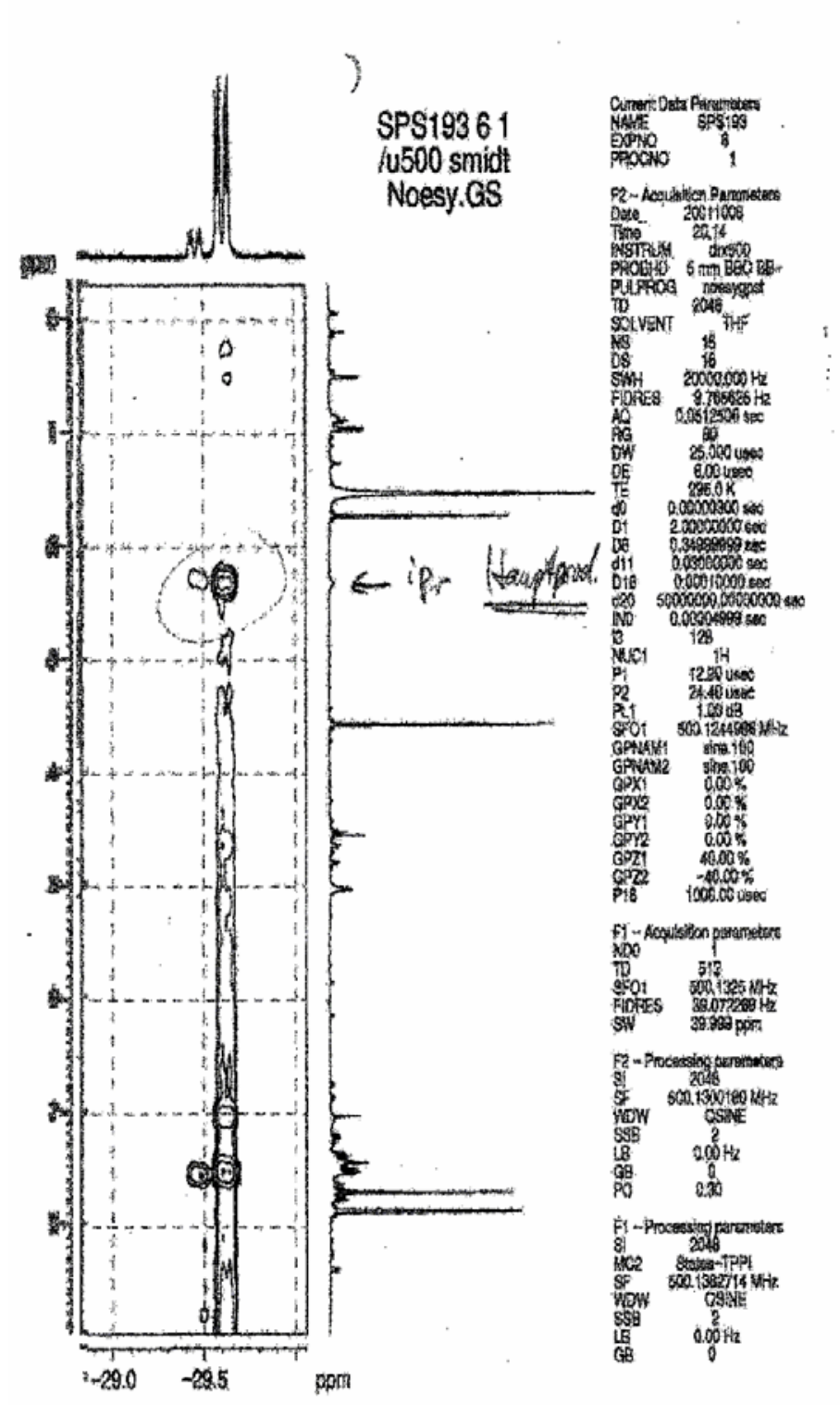


${ }^{31} \mathrm{P}\left\{{ }^{1} \mathrm{H}\right\}$ spectra of $\left.\left[(\mathrm{PHOX}) \operatorname{Ir}(\mathrm{H})_{2} \text { (solvent }\right)_{2}\right] \mathrm{BAr}_{\underline{E}} \underline{(\mathbf{C}-2 \text { \& D-2 })}$ (500 MHz; [D8]-THF) at $0^{\circ} \mathrm{C}$

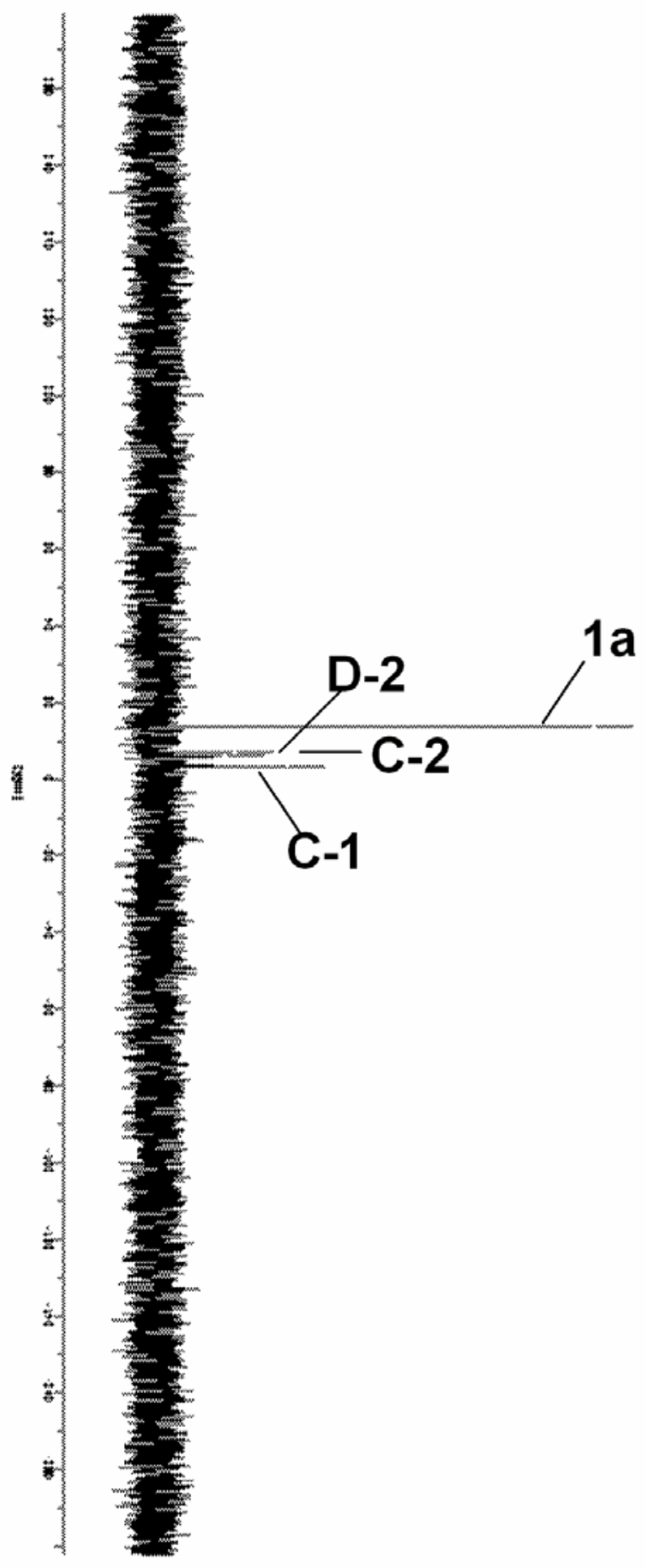


Representative bond lengths and angle for calculated isomers A2-D2

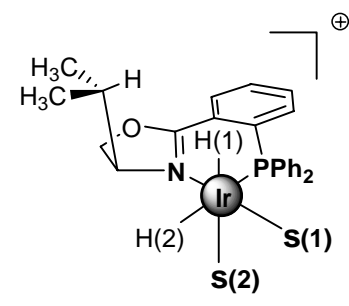

A-2

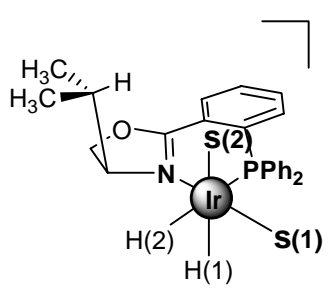

B-2

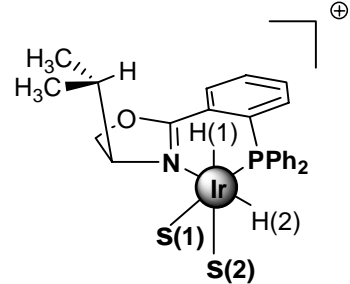

C-2

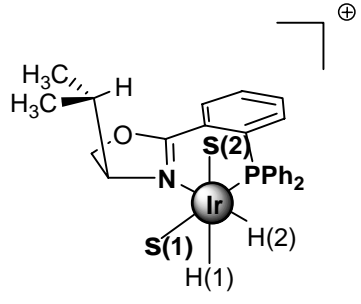

D-2

\begin{tabular}{|c|c|c|c|c|c|c|c|c|}
\hline $\begin{array}{l}\text { solvato- } \\
\text { complex }\end{array}$ & $\begin{array}{c}\text { Rel. E } \\
\text { (kcal/mol) }\end{array}$ & $\begin{array}{r}\text { Ir-P } \\
(\AA)\end{array}$ & $\begin{array}{l}\text { Ir-N } \\
(\AA)\end{array}$ & $\begin{array}{c}\text { N-Ir-P } \\
\left({ }^{\circ}\right)\end{array}$ & $\begin{array}{c}\text { Ir-H(1) } \\
\text { (A) }\end{array}$ & $\begin{array}{c}\operatorname{Ir}-\mathbf{H}(2) \\
(\AA)\end{array}$ & $\begin{array}{c}\text { Ir-S(1) } \\
(\AA)\end{array}$ & $\begin{array}{c}\text { Ir-S(2) } \\
(\AA)\end{array}$ \\
\hline C-2 & - & 2.24 & 2.22 & 87.54 & 1.55 & 1.59 & 2.60 & 2.73 \\
\hline D-2 & +5.0 & 2.23 & 2.20 & 88.86 & 1.54 & 1.59 & 2.57 & 5.96 \\
\hline A-2 & +8.6 & 2.42 & 2.03 & 87.47 & 1.55 & 1.62 & 2.47 & 2.73 \\
\hline B-2 & +14.7 & 2.42 & 2.04 & 88.2 & 1.55 & 1.62 & 2.47 & 2.76 \\
\hline
\end{tabular}

$\mathrm{S}=\mathrm{ClCH}_{3} ; \mathrm{Ir}-(\mathrm{S})=\mathrm{Ir}-\mathrm{Cl}$ distance 